PAPER

An ac susceptibility study of spin dynamics in a super spin glass nanoparticle $\mathrm{La}_{0.7} \mathrm{Ca}_{0.3} \mathrm{MnO}_{3}$ system: simultaneous relaxation processes

To cite this article: M Perovic et al 2013 J. Phys. D: Appl. Phys. 46165001

View the article online for updates and enhancements.
Related content

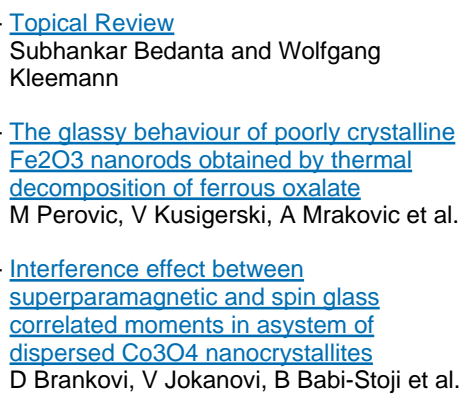

The glassy behaviour of poorly crystalline Fe2O3 nanorods obtained by thermal decomposition of ferrous oxalate M Perovic, V Kusigerski, A Mrakovic et al.

Interference effect between

superparamagnetic and spin glass correlated moments in asystem of dispersed $\mathrm{Co} 3 \mathrm{O} 4$ nanocrystallites

D Brankovi, V Jokanovi, B Babi-Stoji et al.

\section{Recent citations}

- The study of magnetic properties and
$\frac{\text { relaxation processes in } \mathrm{Co} / \mathrm{Au} \text { bimetallic }}{\text { nanoparticles }}$
Pavol Hrubovák et al
- Evidence of superspin-glass behavior in
$\frac{\mathrm{Zn}_{{ }_{5} \mathrm{Ni}_{{ }_{5}} \mathrm{Fe}_{2} \mathrm{O}_{4} \text { nanoparticles }}^{\text {Cristian E Botez et al }}}{\text { - Non-equilibrium magnetic properties of }}$
$\frac{\text { Sm0.43Ca0.57MnO3 nanoparticles }}{\text { V. Markovich et al }}$

\section{HDEN}

Contact Hiden Analytical for further details: w www.HidenAnalytical.com E info@hiden.co.uk

CLICK TO VIEW our product catalogue

\section{Instruments for Advanced Science}
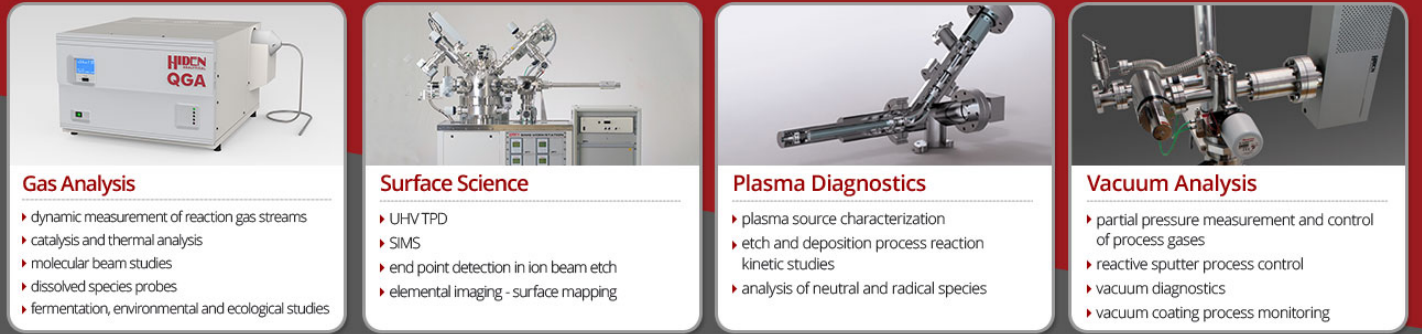

This content was downloaded from IP address 147.91 .1 .45 on $15 / 03 / 2018$ at $12: 33$ 


\title{
An ac susceptibility study of spin dynamics in a super spin glass nanoparticle $\mathrm{La}_{0.7} \mathrm{Ca}_{0.3} \mathrm{MnO}_{3}$ system: simultaneous relaxation processes
}

\author{
M Perovic ${ }^{1}, \mathbf{V}$ Kusigerski $^{1}$, V Spasojevic ${ }^{1}$, A Mrakovic $^{1}$, J Blanusa ${ }^{1}$, \\ $M$ Zentkova $^{2}$ and $M$ Mihalik $^{2}$ \\ ${ }^{1}$ The Vinca Institute, University of Belgrade, PO BOX 522, 11001 Belgrade, Serbia \\ ${ }^{2}$ Institute of Experimental Physics, Slovak Academy of Sciences, 04001 Kosice, Slovak Republic \\ E-mail: mara.perovic@ vinca.rs (M Perovic)
}

Received 16 November 2012, in final form 4 March 2013

Published 27 March 2013

Online at stacks.iop.org/JPhysD/46/165001

\begin{abstract}
In this paper, we present the results of a systematic study of nonequilibrium dynamics in a strongly interacting super spin glass (SSG) nanoparticle $\mathrm{La}_{0.7} \mathrm{Ca}_{0.3} \mathrm{MnO}_{3}$ system by alternating current (ac) susceptibility measurements. Cole-Cole analysis of the obtained data revealed the simultaneous existence of two separated relaxation processes, which can be assigned to the relaxation of different magnetic entities. Along with the expected relaxation of the collective SSG phase, the existence of individual, nonagglomerated particles, which do not take part in the collective phase and relax independently, was proposed. A full dynamical scaling analysis was performed in order to elucidate the nature of the transition to a low-temperature SSG state in the interacting $\mathrm{La}_{0.7} \mathrm{Ca}_{0.3} \mathrm{MnO}_{3}$ nanoparticle sample.
\end{abstract}

\section{Introduction}

Spin dynamics in nanoparticle systems has been a subject of intense research in theoretical and experimental physics since the pioneering study/work of Stoner and Wohlfarth [1]. Understanding of relaxation mechanisms and consequently the possibility of controlling coercivity in nanomaterials opens a number of significant technological applications, especially in the field of information storage, where magnetic nanoscale materials were recognized as promising candidates for a further increase in the density of magnetic storage devices [2]. On the other hand, a number of studies were devoted to the understanding of spin dynamics in magnetic nanoparticle colloidal suspensions [3,4]. They found remarkable applications in biomedicine as contrasts in magnetic resonance imaging, for targeted drug delivery and hyperthermia, as well as biomarkers and biosensors [5, 6].

The degree of interparticle interactions profoundly affects the spin dynamics in a nanoparticle system changing its behaviour from superparamagnetic (SPM) in the case of negligible interactions to collective-spin glass as in the case of strong interactions. The recent experimental and theoretical efforts were directed to the investigation of strongly interacting nanoparticle systems, which exhibit a plethora of unusual magnetic phenomena and whose complex relaxation dynamics is still insufficiently understood.

$\mathrm{La}_{1-x} \mathrm{Ca}_{x} \mathrm{MnO}_{3}$ (LCMO) systems have attracted significant scientific interest in the last few decades due to the variety of observed relaxation phenomena that resemble the behaviour of spin glasses. For the bulk material, the origin of glassy dynamics was found in the frustration of the magnetic ground state, although the nature of frustration remains controversial [7-9]. First and foremost it arises from the competition of super exchange (SE) $\mathrm{Mn}^{3+}-\mathrm{Mn}^{3+}$ interaction, leading to antiferromagnetic (AFM) ordering and double exchange (DE) $\mathrm{Mn}^{3+}-\mathrm{Mn}^{4+}$ mechanism which leads to ferromagnetic (FM) ordering. On the other hand, in low doped bulk manganites frustration is attributed to the formation of a phase-separated state. Phase separation implies the appearance of spatially confined magnetic regions (clusters) of submicrometre size with magnetic coupling different from the surrounding FM background [10-13]. Competing exchange interactions among clusters 
lead to additional frustration, which results in the appearance of spin glass like dynamics at low temperatures [14]. Such a state is usually referred to as a cluster spin glass state. However, origin of the frustration in manganites appeared to be different even in the same material in different intervals of composition or in the samples prepared by different methods.

Spin glass like dynamics was also observed in nanoparticle LCMO systems $[15,16,18,19]$. In order to understand the origin of such behaviour in a nanoparticle material, one has to recognize all the parameters that affect the relaxation dynamics of nanoparticle magnetic moments. The latter depends on the magnetic anisotropy, particle size distribution, the nature and strength of interparticle interactions and surface effects. Also, intraparticle spin disorder due to the finite size effects plays a significant role. In order to elucidate the importance of the above-mentioned factors and understand the nature of relaxation processes in spin glass like nanoparticle systems, we performed a systematic study of nonequilibrium dynamics on the interacting nanoparticle $\mathrm{La}_{0.7} \mathrm{Ca}_{0.3} \mathrm{MnO}_{3}$ sample [17-19].

In this paper, we report on the results of the low-temperature time-dependent phenomena, which were investigated by means of alternating current (ac) susceptibility measurements as a continuation of the magnetic relaxation behaviour study on the same sample. The ac susceptibility data are discussed in terms of Cole-Cole formalism, which enabled further analysis of the experimentally observed relaxation processes. In addition, the dynamic scaling model developed for spin glasses was applied in order to investigate the nature of the transition to a low-temperature super spin glass (SSG) state in the interacting nanoparticle LCMO system.

Nonequilibrium dynamics of SSG nanoparticle systems shows certain differences from the one of archetypal spin glasses regarding anisotropy and different time scales (much longer microscopic flip time of a magnetic moment than of an atomic spin [20]). The experimental time window of ac susceptibility measurements makes this technique a powerful tool for investigation of dynamics in interacting nanoparticle systems.

\section{Previous results and experimental details}

The nanoparticle $\mathrm{La}_{0.7} \mathrm{Ca}_{0.3} \mathrm{MnO}_{3}$ sample was obtained by a mechanochemical milling procedure using the micro mill Fritsch Pulversite 7. The synthetic route was performed under ambient conditions starting from corresponding oxides $\left(\mathrm{La}_{2} \mathrm{O}_{3}, \mathrm{MnO}_{2}\right)$ and $\mathrm{CaCO}_{3}$ as precursors. A detailed report on the mechanochemical synthesis of nanoparticle $\mathrm{La}_{0.7} \mathrm{Ca}_{0.3} \mathrm{MnO}_{3}$ as well as the systematic investigation of its structural and magnetic properties can be found in our previous paper [17]. Transmission electron microscopy (TEM) confirmed the existence of large grains formed by agglomeration of well-crystallized single nanoparticles. An analysis of the recorded micrographs showed that nanoparticles were predominantly spherical in shape with 5-15 nm size range, and with an average particle diameter of $9 \mathrm{~nm}$. A nanoparticle system with such morphology and degree of agglomeration exhibits significant dipolar interactions between nanoparticle magnetic moments. Magnetic measurements showed that the system exhibits cooperative slow dynamics below $T_{\mathrm{SSG}} \approx 47 \mathrm{~K}$ due to a frustration caused by interparticle interactions [18]. Such dynamics was evident in low-temperature experiments suggesting the formation of SSG low-temperature state. The SSG behaviour was confirmed by revealing typical experimental signatures (fingerprints) of cooperative behaviour through direct current (dc) zero field cooled (ZFC) and field cooled (FC) memory effects, as well as ac susceptibility measurements [18]. Magnetic relaxation dynamics was investigated through the set of ZFC and thermoremanent (TRM) magnetization relaxation measurements, which confirmed that the low-temperature SSG dynamics was strongly influenced by the interparticle dipolar interactions [19].

In this paper, the dynamics of a strongly interacting LCMO nanoparticle system as well as crossover from paramagnetic response to a slow spin glass like dynamics is further probed by ac susceptibility experiments. The measurements of the linear ac magnetic susceptibility were made on a commercial superconducting quantum interference device (SQUID) magnetometer MPMS XL-5 in the temperature range $2-80 \mathrm{~K}$ at various $\omega / 2 \pi$ frequencies ranging from 0.5 to $808 \mathrm{~Hz}$. Both in-phase $\chi^{\prime}$ and out-of-phase $\chi^{\prime \prime}$ susceptibility components were measured in the zero dc field, by applying an oscillating field of small amplitude $\mu_{0} h_{\mathrm{ac}}=0.25 \mathrm{mT}$ in order to avoid nonlinear magnetization effects and to maintain the system ground state virtually unaffected. Before setting the ac field, the ultra low field option was used to reduce the remanent field in the superconducting magnet below $0.01 \mathrm{Oe}$.

\section{Results and discussion}

Both in-phase $\chi^{\prime}$ and out-of-phase $\chi^{\prime \prime}$ susceptibility components show well-defined maxima at different temperatures, figure 1. The peak position in the $\chi^{\prime}$ susceptibility curve is found at $47 \mathrm{~K}$ for $\omega / 2 \pi=0.5 \mathrm{~Hz}$ and shifts towards higher temperatures with increasing frequency of the oscillating field. Such a shift of the $\chi^{\prime}$ peak position is characteristic of most of the spin glass [21-23] and SPM nanoparticle systems [24]. The out-of-phase susceptibility curves $\chi^{\prime \prime}(T)$ show different shapes in the low-frequency (LF) region $(0.5-20 \mathrm{~Hz})$ and in the high-frequency $(\mathrm{HF})$ region $(80-808 \mathrm{~Hz})$, with maxima at $28 \mathrm{~K}$ and $34 \mathrm{~K}$, respectively. The peak position shifts with increasing frequency to higher values only for the $\chi^{\prime \prime}$ curves measured in the LF range $(0.5-20 \mathrm{~Hz})$.

Further analysis will focus on the out-of-phase susceptibility $\chi^{\prime \prime}$ because it singles out the processes with relaxation time $t \approx 1 / \omega$ and hence is a direct indication of the spectral distribution of the relaxation processes. In contrast, the in-phase susceptibility $\chi^{\prime}$ integrates over all relaxation processes which are fast compared with $t \approx 1 / \omega$ and, therefore, is rather inconspicuous [25].

\subsection{Cole-Cole analysis}

The ac susceptibility data were analysed for different temperatures in terms of the Cole-Cole formalism, which enables the description of spin dynamics at a given temperature 

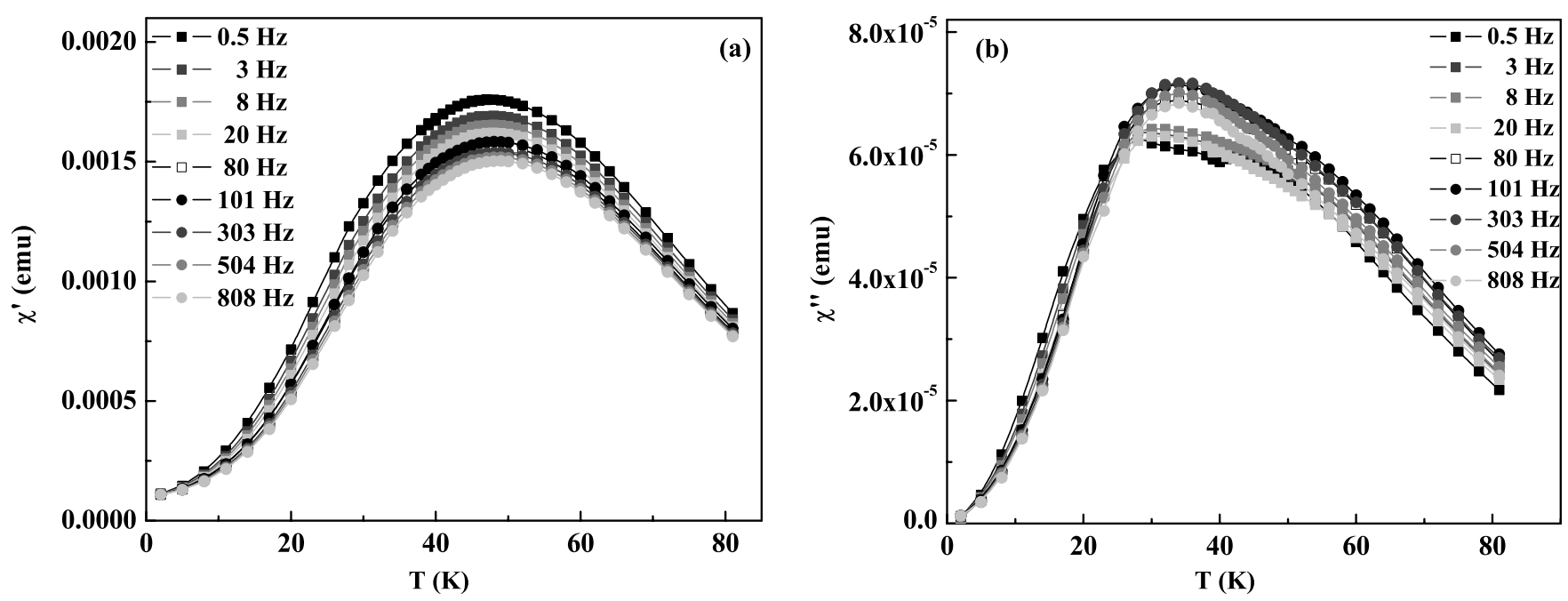

Figure 1. In-phase $\chi^{\prime}(a)$ and out-of-phase $\chi^{\prime \prime}(b)$ components of ac susceptibility as a function of temperature for different frequencies of the oscillating ac field.

and extraction of relaxation time distribution [22]. It represents the magnetic analogue of the Debye theory, originally developed to describe the low frequency dependence of the dielectric constant in polar liquids. The thermodynamic model for relaxation of a magnetic system in an oscillating magnetic field was first derived by Casimir and du Pre [24,26]. It considers energy exchange between magnetic moments and lattice oscillations, i.e. a spin-lattice relaxation mechanism, but can be adequately used to describe other relaxation processes. This model was further modified in order to take into account the presence of more than one relaxation time, or the distribution of relaxation times, resulting in the following expression for magnetic susceptibility [27]:

$$
\chi(\omega)=\chi_{\mathrm{s}}+\frac{\chi_{0}-\chi_{\mathrm{s}}}{1+\left(\mathrm{i} \omega \tau_{\mathrm{c}}\right)^{1-\alpha}}
$$

where $\tau_{\mathrm{c}}$ represents the characteristic relaxation time while the parameter $\alpha$ ranges from 0 to 1 . The parameter $\chi_{0}$ represents the isothermal susceptibility (susceptibility in the limit of zero frequency of the applied magnetic field) [28]. Actually, if processes relaxing magnetization towards equilibrium are very rapid in comparison with oscillations of the magnetic field then magnetization is able to follow the applied field almost instantaneously. In this case, the system responds at a constant temperature ('isothermal' response). On the other hand, if the frequency of the applied field is very high in comparison with the system relaxation rate then the magnetization cannot follow the applied field. In such a case, the system response is 'adiabatic' suggesting the response of spins which are isolated from the external field $[28,29]$. In that sense, $\chi_{\mathrm{s}}$ is the adiabatic susceptibility (susceptibility at a high frequency of the applied oscillating field).

Essentially, this model introduces a distribution function of the relaxation times $g(\tau)$ as

$$
g(\tau)=\frac{1}{2 \pi} \frac{\sin (\alpha \pi)}{\cosh \left[(1-\alpha) \ln \left(\frac{\tau}{\tau_{c}}\right)\right]-\cos (\alpha \pi)}
$$

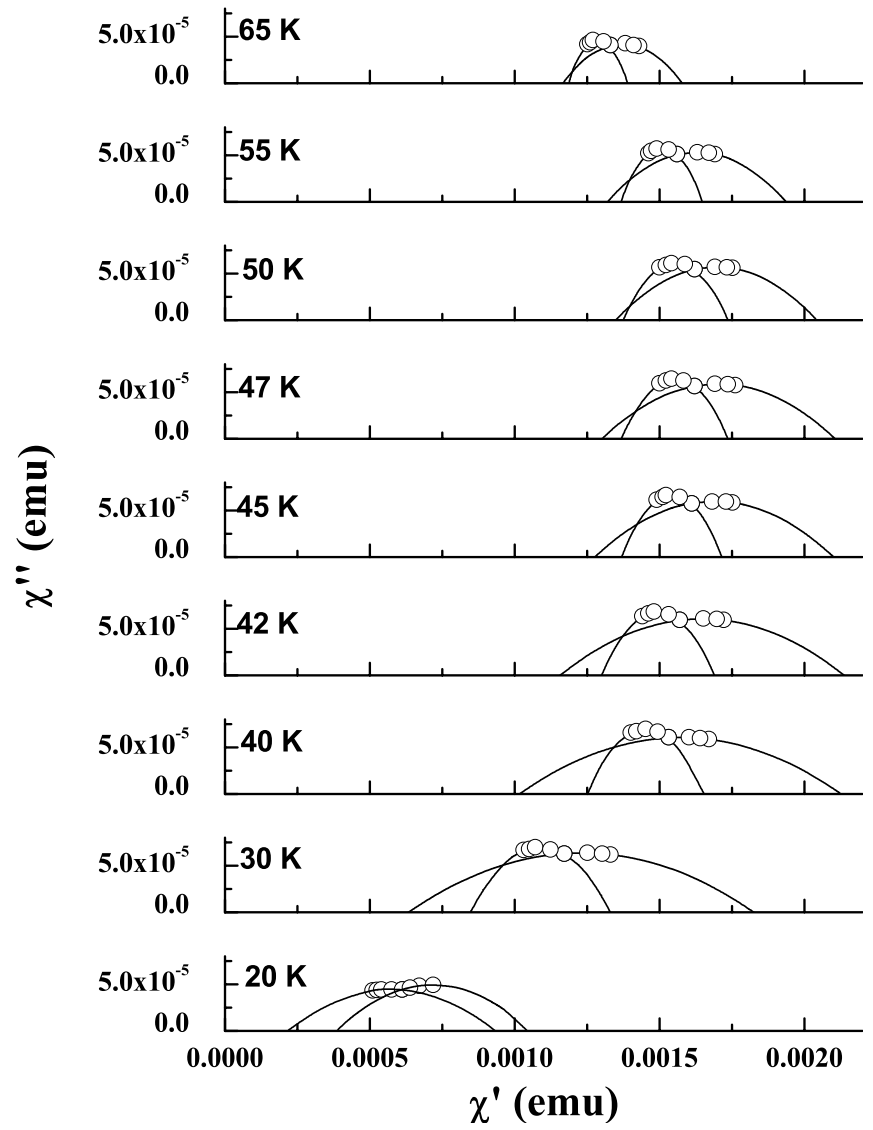

Figure 2. Cole-Cole plots at various temperatures. Dots represent experimental $\chi^{\prime \prime}$ versus $\chi^{\prime}$ data for different frequencies of ac field. Lines denote fits of experimental data to equation $(3 c)$.

where $\alpha$ represents the width of the relaxation time distribution [22]. For monodispersive ensemble of noninteracting SPM particles (Debye model) the parameter $\alpha=0$. On the other hand, $\alpha=1$ corresponds to an infinitely wide distribution.

Decomposing of equation (1) gives the expressions for in-phase $\chi^{\prime}(\omega)$ and out-of-phase $\chi^{\prime \prime}(\omega)$ susceptibilities 


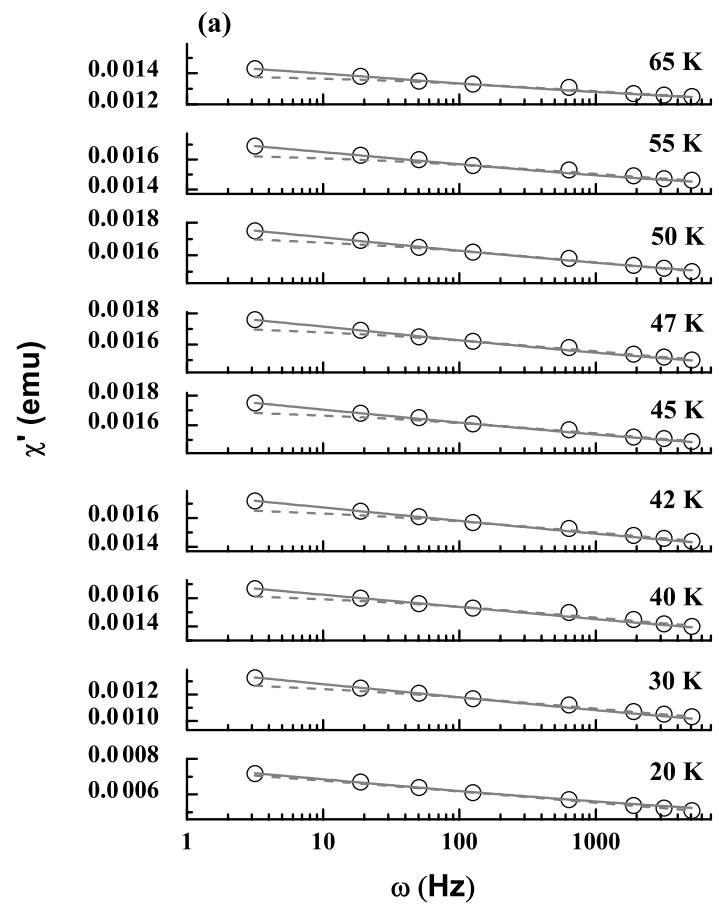

(b)

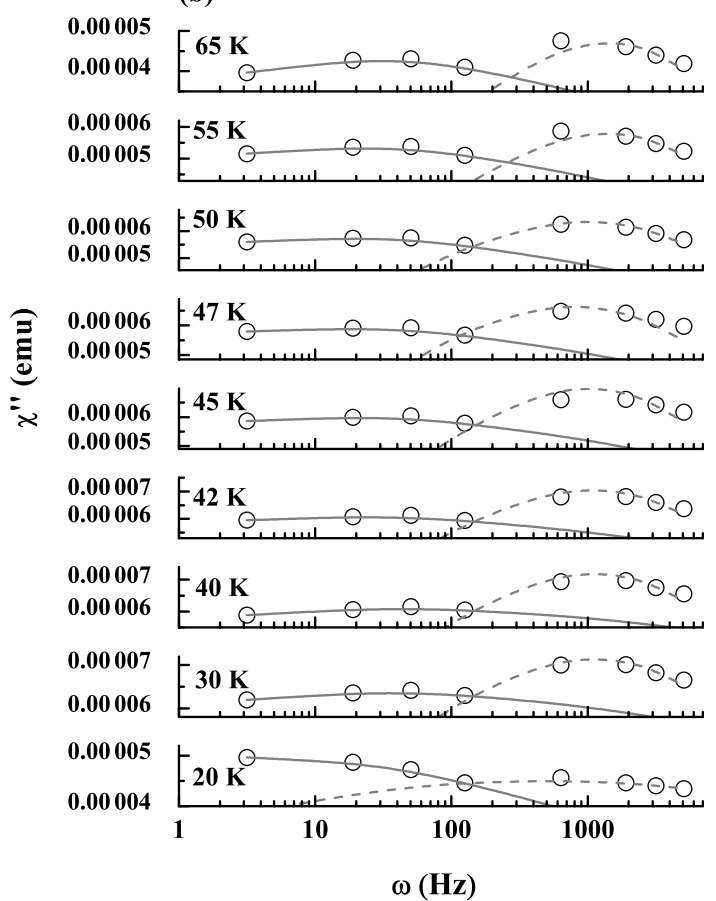

Figure 3. Frequency dependence of the $(a) \chi^{\prime}$ component and $(b) \chi^{\prime \prime}$ component at various temperatures. Dots represent experimental data, while lines denote fits of experimental data to equations $(3 a)$ and $(3 b)$. Fits in LF and HF regions are represented by solid and dashed lines, respectively. $X$-axis is represented in logarithmic scale.

\section{$[22,30,31]:$}

$$
\begin{aligned}
\chi^{\prime}(\omega) & =\chi_{\mathrm{s}}+\frac{\chi_{0}-\chi_{\mathrm{s}}}{2} \\
\times & \left(1-\frac{\sinh \left[(1-\alpha) \ln \left(\omega \tau_{\mathrm{c}}\right)\right]}{\cosh \left[(1-\alpha) \ln \left(\omega \tau_{\mathrm{c}}\right)\right]+\cos \left[\left(\frac{1}{2}\right)(1-\alpha) \pi\right]}\right)
\end{aligned}
$$

$$
\begin{aligned}
\chi^{\prime \prime}(\omega) & =\frac{\chi_{0}-\chi_{\mathrm{s}}}{2} \\
\times & \left(\frac{\sin \left[\left(\frac{1}{2}\right)(1-\alpha) \pi\right]}{\cosh \left[(1-\alpha) \ln \left(\omega \tau_{\mathrm{c}}\right)\right]+\cos \left[\left(\frac{1}{2}\right)(1-\alpha) \pi\right]}\right)
\end{aligned}
$$

The experimental data were plotted in the complex plane as $\chi^{\prime \prime}$ versus $\chi^{\prime}$ for selected temperatures and fitted to the equation of a circular arc (Cole-Cole or Argand plot):

$$
\begin{aligned}
& \chi^{\prime \prime}\left(\chi^{\prime}\right)=-\frac{\chi_{0}-\chi_{\mathrm{s}}}{2 \tan \left[(1-\propto) \frac{\pi}{2}\right]} \\
& +\sqrt{\left(\chi^{\prime}-\chi_{\mathrm{s}}\right)\left(\chi_{0}-\chi^{\prime}\right)+\frac{\left(\chi_{0}-\chi_{\mathrm{s}}\right)^{2}}{4 \tan ^{2}\left[(1-\alpha) \frac{\pi}{2}\right]}}
\end{aligned}
$$

with three adjustable parameters $\chi_{0}, \chi_{\mathrm{s}}$ and $(1-\alpha)$. ColeCole plots obtained in the fitting procedure for different temperatures are depicted in figure 2. Prominent flatness and/or distortion of the displayed semicircles are expected to be found in polydispersive systems, such as nanoparticle systems with a wide distribution of particle sizes or some spin glasses with a broad distribution of relaxation times due to collective behaviour [21].

The parameter $\tau_{\mathrm{c}}$ was calculated afterwards from the fit of $\chi^{\prime}(\omega)$ and $\chi^{\prime \prime}(\omega)$ dependences (figure 3 ) to the equations (3a) and $(3 b)$. During this fitting procedure, previously determined parameters $\chi_{0}$ and $\chi_{\mathrm{s}}$ were kept constant, while only $\tau_{\mathrm{c}}$ and $(1-\alpha)$ were allowed to vary. The whole set of parameters was obtained using this two-step fitting procedure.

Cole-Cole plots at various temperatures revealed the presence of a composite curve deviating from the usual single semicircle (indicative of a single relaxation time [32]), figure 2. Experimentally obtained $\chi^{\prime \prime}\left(\chi^{\prime}\right)$ data showed two distinct flattened semicircles, one in the LF, and another in the HF region. Each of the two broad overlapping arcs was fitted separately according to equation $(3 c)$. It appears that the distinction observed in the $\chi^{\prime \prime}(T)$ curves measured in the LF and $\mathrm{HF}$ regions (figure 1) reflects the simultaneous existence of two distinct relaxation processes.

This assumption was further confirmed through the analysis of the fitting parameters, which revealed different characteristic relaxation times $\tau_{\mathrm{c}}$ for two processes. Temperature dependences of the characteristic relaxation time for both processes are depicted in figure 4 . In both cases, characteristic relaxation time $\tau_{\mathrm{c}}$ shows anomaly/jump at $T=47 \mathrm{~K}$, as might be expected on the basis of conventional critical slowing down of the spin relaxation time according to the dynamical scaling hypothesis near a phase transition temperature [21]. The parameter $\tau_{\mathrm{c}}$ increases rapidly with decreasing temperature starting from $\approx 30 \mathrm{~K}$. For the process observed at low frequencies $\tau_{\mathrm{c}}$ changes from $10^{-2} \mathrm{~s}$ to $10^{3} \mathrm{~s}$, while for the HF process $\tau_{\mathrm{c}}$ changes from $10^{-4} \mathrm{~s}$ at high temperatures to $10^{-2} \mathrm{~s}$ at low temperatures.

The behaviour of $\alpha$ parameter appears to be different in the LF and HF cases, figure 5. In the LF susceptibility curves, the behaviour of $\alpha$ is similar to that reported for different spin glass systems [23,32-36]. It varies slowly, almost linearly 

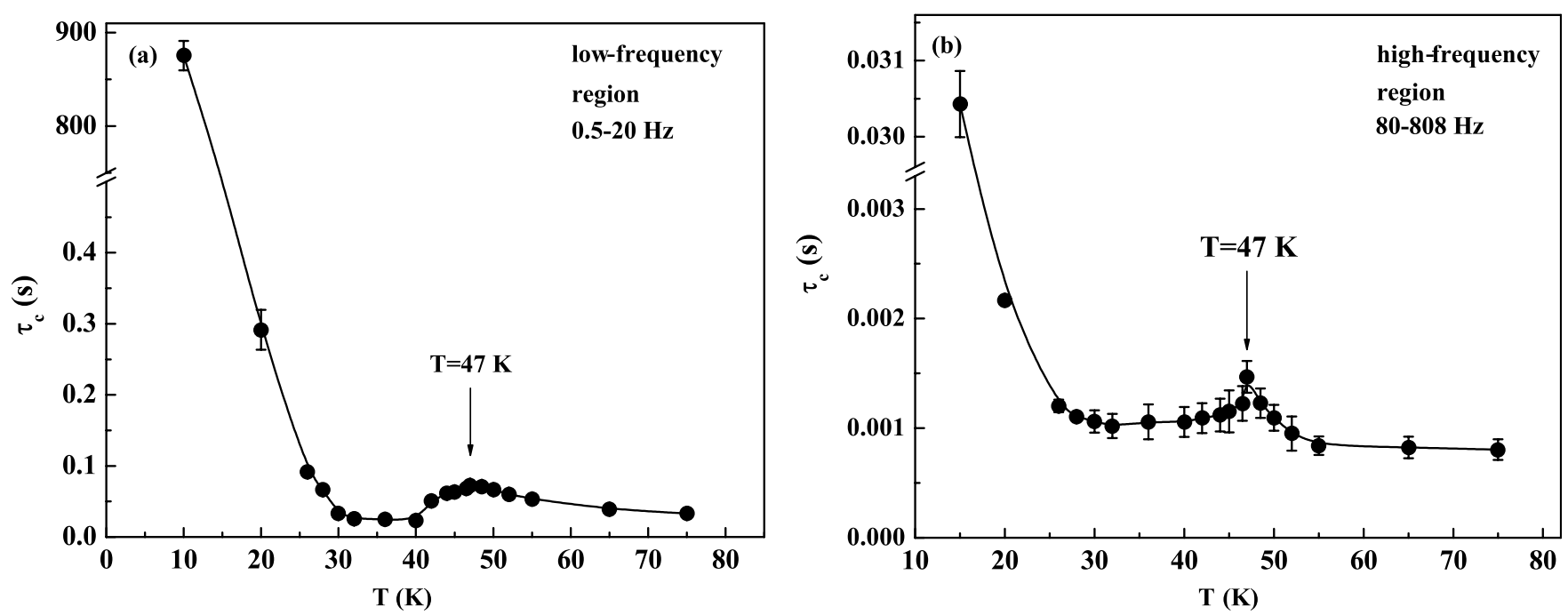

Figure 4. Temperature dependence of the characteristic relaxation time $\tau_{\mathrm{c}}$ for the relaxation process observed in the $(a) \mathrm{LF}$ and $(b) \mathrm{HF}$ regions. Only the absolute errors larger than the size of data symbols are denoted by error bars.
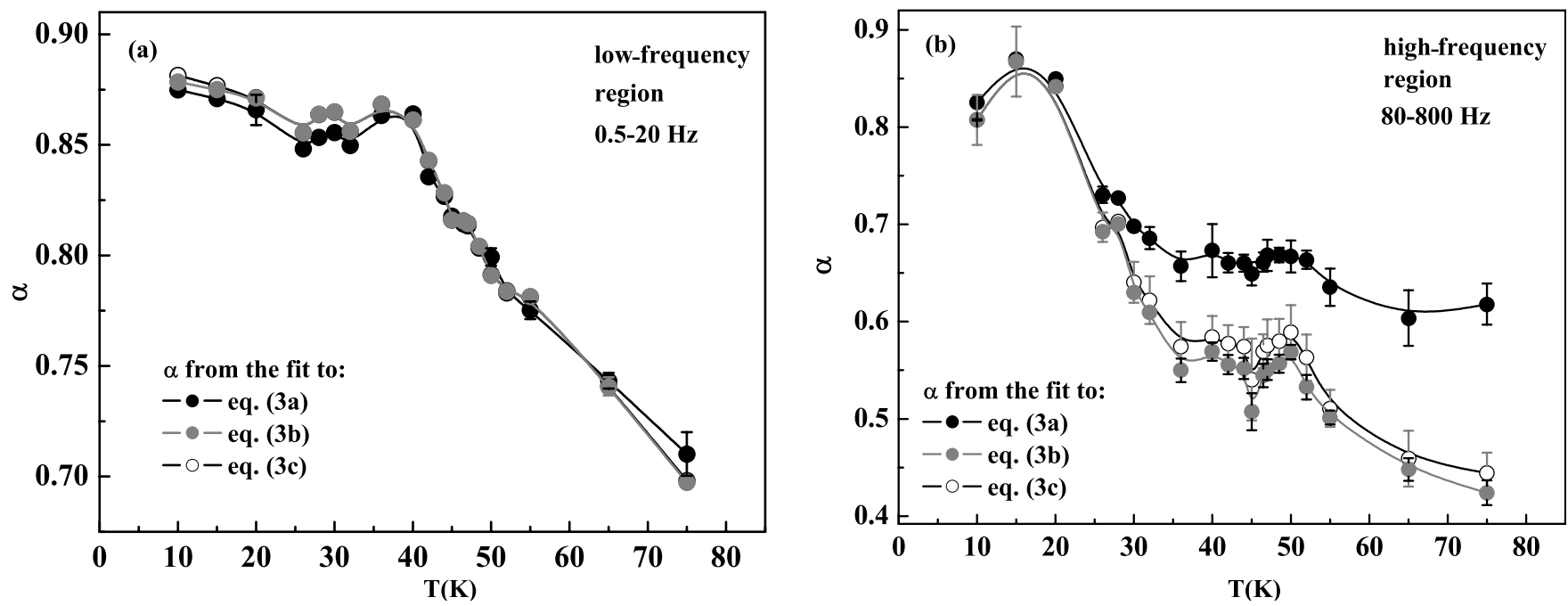

Figure 5. Temperature dependence of parameter $\alpha$ for the relaxation process observed in the (a) LF and (b) HF regions. Values of $\alpha$ were extracted from the fit of experimental data to equations $(3 a)-(3 c)$. Only the absolute errors larger than the size of data symbols are denoted by error bars.

at low temperatures. The slope of the $\alpha(T)$ curve becomes more pronounced for temperatures above $\approx 30 \mathrm{~K}$. The fitting procedure performed in the fine temperature step revealed irregular change of parameter $\alpha$ for temperatures around $30 \mathrm{~K}$. As $\alpha$ can be understood as the width of the relaxation time distribution, it is clear that in the LF case we deal with an extremely broad distribution $(\alpha \approx 0.85)$ at low temperatures (below $T \approx 30 \mathrm{~K}$ ), which gradually narrows down to the value $\alpha \approx 0.7$ with the increase in temperature. Such behaviour is indicative of a spin glass like freezing process [21]. Although the origin of the slope change in the $\alpha(T)$ curves at $T \approx 30 \mathrm{~K}$ is still unclear, it is noticeable that it appears at the temperature close to the temperature of maximum in the $\chi^{\prime \prime}(T)$ curve for low frequencies.

In the HF case, the width of the relaxation time distribution narrows more rapidly with the increase in temperature, from $\alpha \approx 0.85$ at low temperatures to the value $\alpha \approx 0.4$ obtained at the highest measured temperature. The gradual change of distribution width with temperature can be related to progressive blocking of particle moments. The pronounced anomaly at $47 \mathrm{~K}$ is visible only in the HF case.

Parameters $\chi_{\mathrm{s}}$ and $\chi_{0}$ show a pronounced peak at $47 \mathrm{~K}$, with similar temperature behaviour in both LF and HF cases, figure 6.

The behaviour of both $\tau_{\mathrm{c}}$ and $\alpha$ parameters gives clear evidence that at least two relaxation mechanisms govern the slow relaxation of the sample. Two relaxation pathways can be assigned to the relaxation of different magnetic entities. It may be expected that susceptibility coming from the total magnetization in a magnetic domain will be substantial rather at low frequencies [24]. This kind of relaxation process in our LCMO sample can be assigned to the clusters of nanoparticles whose existence was confirmed by TEM analysis [17]. Its characteristic feature is slow magnetic relaxation ( $\tau_{\mathrm{c}} \approx 10^{-2} \mathrm{~s}$ at the highest measured temperature) with a change in characteristic relaxation time of five orders of magnitude in the measuring temperature interval. On the other hand, it is obvious that there are magnetic entities, which 

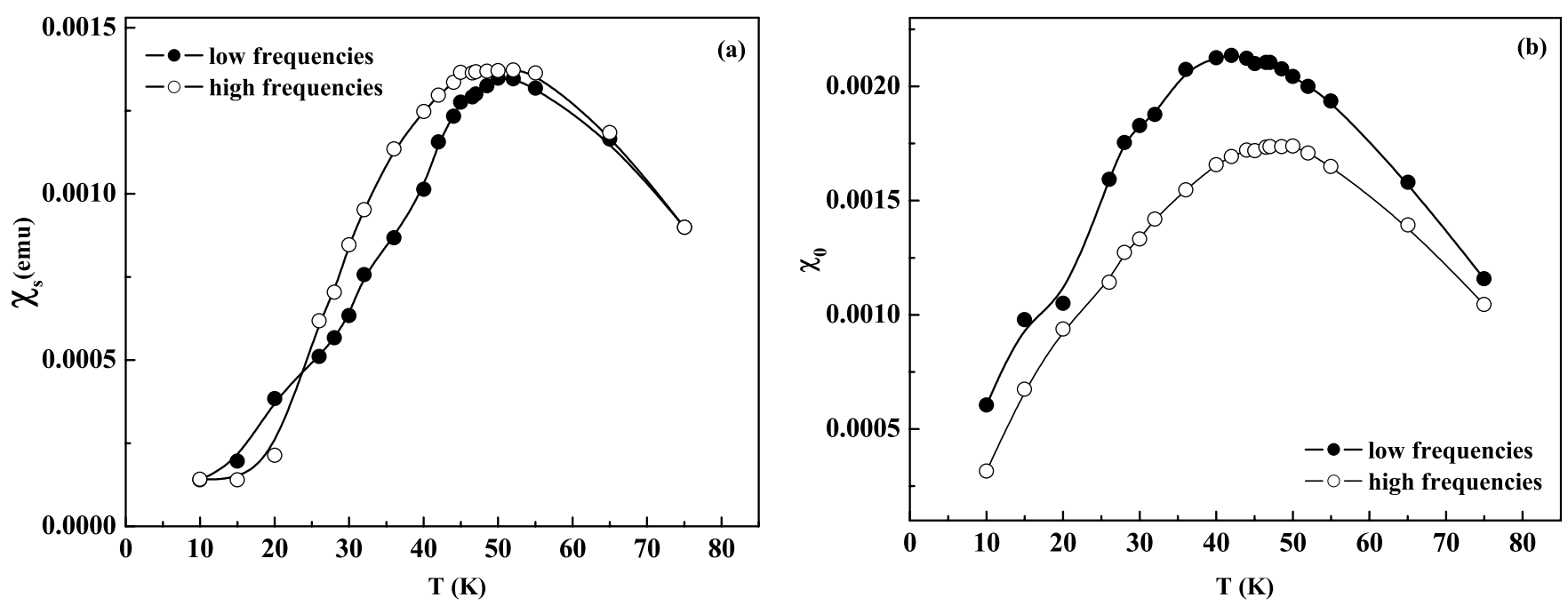

Figure 6. Temperature dependence of the $(a) \chi_{\mathrm{s}}$ and $(b) \chi_{0}$ parameters for the relaxation processes observed in the LF and HF regions. Mean relative errors are as follows: $(a)$ of $\chi_{\mathrm{s}}$ parameter, $0.34 \%$ for the LF case and $1.4 \%$ for the HF case; $(b)$ of $\chi_{0}$ parameter, $0.22 \%$ for the LF case and $0.85 \%$ in the HF case.

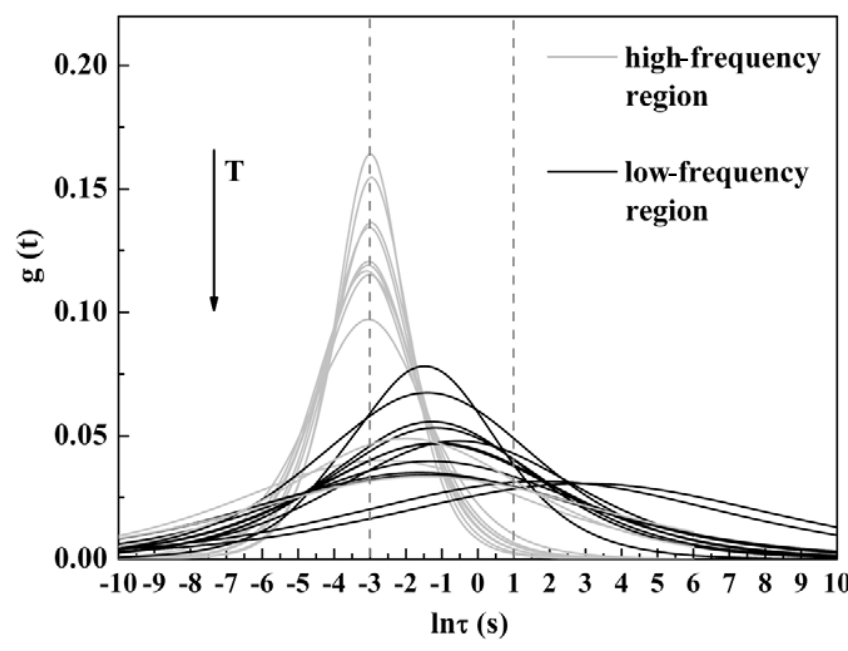

Figure 7. Distribution of relaxation times at different temperatures for two relaxation processes observed in the LF (black lines) and HF (grey lines) regions. Our experimental time window is contoured with dashed lines.

respond easily to the ac field even at high frequencies, as their equilibrium state can be easily disturbed. They exhibit faster magnetic relaxation $\left(10^{-4} \mathrm{~s}<\tau_{\mathrm{c}}<10^{-2} \mathrm{~s}\right)$ in the measured temperature interval. We attribute these magnetic entities to nonagglomerated individual particles, which do not feel the strong local dipolar field of the surrounding moments. Consequently, the relaxation of their magnetic moments is not hierarchically constrained. In addition, the peak in the HF $\chi^{\prime \prime}(T)$ curves does not shift with the increase in frequency, suggesting that those individual particles behave as entities with bulk-like properties.

With the known values of $\alpha$ and $\tau_{\mathrm{c}}$ parameters, by applying equation (2), it was straightforward to calculate the distribution of relaxation times $g(\tau)$ for both processes at different temperatures, figure 7.

As the temperature decreases, the distribution profile for the relaxation process observed in the LF region broadens and the position of the profile continuously moves towards longer relaxation times, thus indicating constant slowing down of the most probable relaxation time $\tau_{\mathrm{c}}$. A more pronounced increase in the mean relaxation time happens upon cooling down through the freezing temperature. Temperature-dependent distribution width can be expected in SSG nanoparticle systems, in which the correlation length and the corresponding relaxation times of interacting magnetic moments increase with decreasing temperature. In any case, slowing down of the characteristic relaxation time in our LCMO system is less drastic than in the archetypal spin glass systems, where dramatic slowing down of the mean relaxation time upon cooling (even for 16 orders of magnitude) through the freezing temperature was reported for several systems [23, 24, 37]. The behaviour of the distribution of relaxation times in our case suggests that both entities experience additional slowing down at temperatures below approximately $30 \mathrm{~K}$.

\subsection{Dynamical scaling}

Ageing and nonequilibrium dynamics previously reported for the LCMO sample $[18,19]$ indicate, but does not provide decisive evidence for a thermodynamic phase transition at $T_{\mathrm{g}}$ to a low-temperature SSG state. Regarding that, we employed a dynamical scaling of ac susceptibility in order to investigate the nature of transition to the SSG state. Being an independent approach, it is conventionally used as supporting evidence for the critical behaviour at the spin glass transition [20].

In a full dynamical scaling analysis, the imaginary component of the dynamic susceptibility measured at various frequencies $f=\omega / 2 \pi$ is scaled according to [38-40]

$$
\frac{\chi^{\prime \prime}(T, \omega)}{\chi_{\mathrm{eq}}(T)}=\varepsilon^{\beta} H\left(\omega \tau_{\mathrm{c}}\right), \quad T>T_{\mathrm{g}}
$$

where $\chi_{\text {eq }}$ is the equilibrium susceptibility in the limit $\omega \rightarrow 0$, while $\varepsilon$ denotes the reduced temperature $\varepsilon=\left(1-\left(T / T_{\mathrm{g}}\right)\right)$. Data for various frequencies should collapse onto the master 

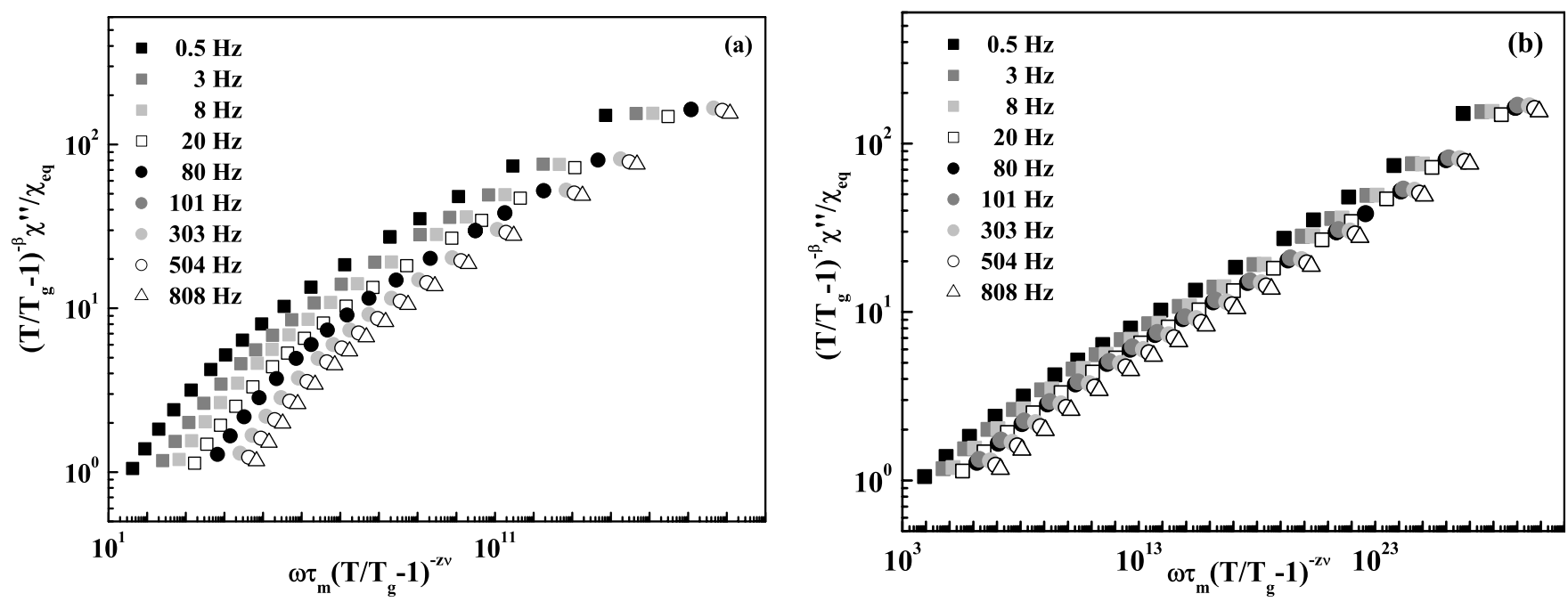

Figure 8. Dynamical scaling of experimentally obtained out-of-phase susceptibility data $\chi^{\prime \prime}$ according to equation (4) with microscopic time scale $\tau_{\mathrm{m}}$ : $(a)$ temperature independent; $(b)$ approximated by the Néel-Arrhenius expression.

curve expressed by the $H\left(\omega \tau_{\mathrm{c}}\right)$ function. $\tau_{\mathrm{c}}$ represents the critical correlation time related to the spin-spin coherence length $\xi$ via exponent $z$ as $\tau_{\mathrm{c}} \propto \tau_{\mathrm{m}} \xi^{z} \propto \varepsilon^{-z \nu}$, where $\tau_{\mathrm{m}}$ is a microscopic time scale. For spin glasses $\tau_{\mathrm{m}} \approx 10^{-13} \mathrm{~s}$ is the fluctuation time of an atomic moment, while for nanoparticles it can be assigned to the SPM relaxation time of a single particle of average size and approximated by the Néel-Arrhenius expression $\tau_{\mathrm{m}}=\tau_{0} \exp \left(E / k_{\mathrm{B}} T\right)$.

Experimentally obtained out-of-phase susceptibility data $\chi^{\prime \prime}$ were scaled according to equation (4) by plotting $\left(\left(T / T_{\mathrm{g}}\right)-1\right)^{-\beta} \frac{\chi^{\prime \prime}}{\chi_{\mathrm{eq}}}$ versus $\omega \tau_{\mathrm{c}}$, figure 8 . The function $\chi_{\mathrm{eq}}$ is approximated by the Curie-Weiss expression $\chi_{\mathrm{eq}}=\frac{\chi_{0}}{T-T_{0}}$, where parameters $\chi_{0}=0.00157 \mathrm{emu}$ and $T_{0}=136 \mathrm{~K}$ are obtained by the fit of the LF in-phase susceptibility $\chi^{\prime}(T)$ data in the high-temperature region $(T>150 \mathrm{~K})$. The first attempt of dynamical scaling with $\tau_{\mathrm{c}} \propto \tau_{\mathrm{m}} \varepsilon^{-z v}$ and the assumption that the microscopic flip time $\tau_{m}$ is temperature independent (as in archetypal spin glasses) failed to scale all curves onto a single master curve, figure $8(a)$. However, with an assumption of Néel-Arrhenius temperature dependence for $\tau_{\mathrm{m}}$, i.e. $\tau_{\mathrm{c}} \propto \tau_{\mathrm{m}} \varepsilon^{-z \nu} \propto \tau_{0} \exp \left(E / k_{\mathrm{B}} T\right) \varepsilon^{-z \nu}$, the scaling was significantly improved, figure $8(b)$. The trial value for $E \sim 10^{2} \mathrm{~K}$ was previously obtained as the anisotropy energy barrier of an average-sized nanoparticle in our sample [17]. The most satisfactory collapse of data is obtained for the following choice of consistent parameter set: $T_{\mathrm{g}}=47 \mathrm{~K}$, $z \nu=8, \beta=1.0$, where the $z \nu^{3}$ and $\beta$ values comply with the analysis on SSG systems [20].

Improved scaling in the case of temperature-dependent microscopic flip time implies that satisfactorily good collapsing of curves could be obtained by a more suitable choice of $\tau_{\mathrm{m}}$ temperature dependence. However, the performed dynamical scaling analysis cannot undoubtedly confirm that the LCMO nanoparticle system exhibits a

3 The used $z v$ value is close to the value $z v=7.4$ (1) obtained from the power law fit of $\chi^{\prime}(T)$ experimental data in [18] where the driving amplitude of the ac field was higher, $\mu_{0} h_{\mathrm{ac}}=0.4 \mathrm{mT}$. In addition, the power law form in [18] assumed the $\tau_{\mathrm{m}}=\tau_{0}=$ const condition thermodynamic phase transition, although it clearly exhibits spin glass dynamics. A static scaling analysis, as performed in [41], should provide a crucial tool to disclose a possible spin glass phase transition.

There is a consensus that full dynamic scaling would reveal a phase transition to a low-temperature SSG state only if the single particle contribution to $\chi^{\prime \prime}$ is vanishingly small for $T>T_{\mathrm{g}}$, i.e. if the slow dynamics is due to collective behaviour. This condition is fulfilled only for strongly interacting nanoparticle systems with a narrow anisotropy barrier distribution [42]. Consequently, the existence of nonagglomerated individual nanoparticles, which do not exhibit collective behaviour, may mask the underlying phase transition. Their contribution to the measured susceptibility has been clearly observed in HF ac susceptibility measurements.

\section{Conclusion}

The nonequilibrium dynamics in the low-temperature SSG state of an interacting LCMO nanoparticle sample was found to strongly resemble the corresponding spin glass dynamics. However, certain differences compared with the archetypal spin glasses were observed. Contrary to temperatureindependent monodispersed relaxation times of atomic spins in spin glasses, Cole-Cole analysis revealed a wide distribution of relaxation times and its temperature dependence. Two parallel relaxation pathways were experimentally observed and the possible mechanism for such relaxation effects was suggested. Along with the expected relaxation of the collective SSG phase, the existence of individual, nonagglomerated particles, which do not take part in the collective phase and relax independently, was proposed. The nature of the transition to the low-temperature SSG state was discussed in terms of full dynamical scaling. Although interacting LCMO nanoparticles clearly exhibit spin glass dynamics, crucial evidence for the existence of thermodynamic phase transition is still missing. 


\section{Acknowledgments}

This work was supported by the Ministry of Education and Science, Republic of Serbia (Project No III-45015).

\section{References}

[1] Stoner E C and Wohlfarth E P 1948 Phil. Trans. R. Soc. Lond. A 24059

[2] Moser A, Takano K, Margulies D T, Albrecht M, Sonobe Y, Ikeda Y, Sun S and Fullerton E E 2002 J. Phys. D: Appl. Phys. 35 R157

[3] Singh V, Banerjee V and Sharma M 2009 J. Phys. D: Appl. Phys. 42245006

[4] Pankhurst Q A, Connolly J, Jones S K and Dobson J 2003 J. Phys. D: Appl. Phys. 36 R167

[5] Berry C C and Curtis A S G 2003 J. Phys. D: Appl. Phys. 36 R198

[6] Tartaj P, Morales M del P, Veintemillas-Verdaguer S, Gonzalez-Carreno T and Serna C J 2003 J. Phys. D: Appl. Phys. 36 R 182

[7] Dagotto E 2002 Nanoscale Phase Separation and Colossal Magnetoresistence - The Physics of Manganites and Related Compounds (New York: Springer)

[8] Coey J M D, Viret M and von Molnar S 1999 Adv. Phys. 48167

[9] Laiho R, Lahderanta E, Salmien J, Lisunov K G and Zakhvalinskii V S 2001 Phys. Rev. B 63094405

[10] Freitas R S, Ghivelder L, Damay F, Dias F and Cohen L F 2001 Phys. Rev. B 64144404

[11] Levy P, Parisi F, Granja L, Indelicato E and Polla G 2002 Phys. Rev. Lett. 89137001

[12] Hennion M, Moussa F, Biotteau G, Rodriguez-Carvajal J, Pinsard L and Revcolevschi A 1998 Phys. Rev. Lett. 811957

[13] Laiho R, Lisunov K G, Lahderanta E, Petrenko P, Salmien J, Stamov V N and Zakhvalinskii V S 2000 J. Phys.: Condens. Matter 125751

[14] Rivadulla F, Lopez-Quintela M A and Rivas J 2004 Phys. Rev. Lett. 93167206

[15] Markovich V, Fita I, Wasniewski A, Jung G, Mogilyansky D, Puzniak R, Titelman L and Gorodetsky G 2010 Phys. Rev. B 81134440

[16] Karmakar S, Chaudhuri B K, Chan C L and Yang H D 2010 J. Appl. Phys. 108113916

[17] Spasojevic V, Mrakovic A, Perovic M, Kusigerski V and Blanusa J 2011 J. Nanopart. Res. 13763

[18] Perovic M, Mrakovic A, Kusigerski V, Blanusa J and Spasojevic V 2011 J. Nanopart. Res. 136805
[19] Perovic M, Mrakovic A, Kusigerski V, Blanusa J and Spasojevic V 2012 Mater. Chem. Phys. 136196

[20] Jonsson P E 2004 Adv. Chem. Phys. 128191

[21] Mydosh J 1983 Spin Glasses: An Experimental Introduction (London: Taylor and Francis)

[22] Dekker C, Arts A F M, de Wijn H W, Duyneveldt A J and Mydosh J A 1989 Phys. Rev. B 4011243

[23] Dekker C, Arts A F M, de Wijn H W, Duyneveldt A J and Mydosh J A 1988 Phys. Rev. Lett. 611780

[24] Balanda M 2003 Relaxation Phenomena: Liquid Crystals, Magnetic Systems, Polymers, High- $T_{\mathrm{c}}$ Superconductors, Metallic Glasses ed W Haase and S Wrobel (Berlin: Springer)

[25] Maletta H and Zinn W 1989 Spin glasses Handbook on the Physics and Chemistry of Rare Earths vol 12, ed K A Geschneider and L Eyring (Amsterdam: North-Holland)

[26] Casimir H B G and du Pre F K 1938 Physica 5507

[27] Cole K S and Cole R H 1941 J. Chem. Phys. 9341

[28] Levy L P 2000 Magnetism and Superconductivity (Texts and Monographs in Physics) (Berlin: Springer)

[29] Fernandez van Raap M B, Sanchez F H, Rodriguez Torres C E, Casas Ll, Roig A and Molins E 2005 J. Phys.: Condens. Matter 176519

[30] Petracic O, Sahoo S, Binek Ch, Kleemann W, Sousa J B, Cardoso S and Freitas P P 2003 Phase Transit. 76367

[31] Hagiwara M 1998 J. Magn. Magn. Mater. 17789

[32] Wegner L E 1983 Heidelberg Colloquium on Spin Glasses (Berlin: Springer)

[33] Saito T, Suto A and Takenaka S 2004 J. Magn. Magn. Mater. 2721319

[34] Baalbergen J J, Ong T S, van Duyneveldt A J and Verstelle J C 1988 J. Physique Coll. 491065

[35] Brankovic D, Jokanovic V, Babic-Stojic B, Jaglicic Z, Lisjak D and Kojic D 2009 J. Phys.: Condens. Matter 21095303

[36] Huser D, van Duyneveldt A J, Nieuwenhuys G J and Mydosh J M 1986 J. Phys. C: Solid State Phys. 193697

[37] Martinez-Blanco D, Gorria P, Fernandez-Martinez A, Perez M J, Cuello G J and Blanco J A 2011 J. Alloys Compounds 509S S397

[38] Zhou Y, Rigaux C, Mycielski A and Menant M 1989 Phys. Rev. B 408111

[39] Djurberg C, Svedlindh P, Nordblad P, Hansen M F, Bodker F and Morup S 1997 Phys. Rev. Lett. 795154

[40] Bedanta S and Kleemann W 2009 J. Phys. D: Appl. Phys. 42013001

[41] Jonsson T, Svedlindh P and Hansen M F 1998 Phys. Rev. Lett. 813976

[42] Jonsson P, Hansen M F, Svenlindh P and Nordblad P 2001 J. Magn. Magn. Mater. 2261315 